\title{
Synthesis of Substituted Carbazoles via a Vinylic to Aryl Palladium Migration Involving Domino C-H Activations
}

\author{
Jian Zhao and Richard C. Larock* \\ Department of Chemistry, Iowa State University, Ames, Iowa 50011 \\ larock@iastate.edu
}

General Procedures. All ${ }^{1} \mathrm{H}$ and ${ }^{13} \mathrm{C}$ spectra were recorded at $300 \mathrm{MHz}$ and 75.5 MHz respectively. Thin-layer chromatography was performed using commercially prepared 60-mesh silica gel plates (Whatman K6F), and visualization was effected with short wavelength UV light $(254 \mathrm{~nm})$ and a basic $\mathrm{KMnO}_{4}$ solution. High resolution mass spectra were recorded on a Kratos MS50TC double focusing magnetic sector mass spectrometer using EI and $70 \mathrm{eV}$.

Reagents. All reagents were used directly as obtained commercially unless otherwise noted. Anhydrous forms of acetonitrile, DMF, diethyl ether, ethyl acetate, hexanes, and 4,4-dimethyl-2-pentyne were purchased from Lancaster Synthesis, Inc. 3-Iodoaniline, 2(trimethylsilyl)phenyl trifluoromethanesulfonate, cesium fluoride, 1,3-diodobenzene, 1,1'-bis(diphenylphosphino)ferrocene (dppf), sodium tert-butoxide, $p$-toluidine, $p$ anisidine, $p$-chloroaniline, 1-naphthylamine, diphenylacetylene were purchased from Aldrich Chemical Co., Inc. Cesium pivalate was prepared according to the procedure of Campo and Larock. ${ }^{1}$

\section{3-Iodo- $N$-phenylbenzenamine (1)}


This compound is prepared according to the reported procedure. ${ }^{2}{ }^{1} \mathrm{H} \mathrm{NMR}\left(\mathrm{CDCl}_{3}\right) \delta$ $5.67(\mathrm{~s}, 1 \mathrm{H}), 6.94-7.10(\mathrm{~m}, 5 \mathrm{H}), 7.23-7.41(\mathrm{~m}, 4 \mathrm{H}) ;{ }^{13} \mathrm{C} \mathrm{NMR}\left(\mathrm{CDCl}_{3}\right)$ 95.22, 116.53, 119.10, 122.30, 125.86, 129.74, 129.76, 131.05, 142.18, 145.05; IR $\left(\mathrm{CDCl}_{3}\right) 3427,3061$, 3034, $1584 \mathrm{~cm}^{-1}$; HRMS m/z 294.9858 (calcd for $\mathrm{C}_{12} \mathrm{H}_{10} \mathrm{NI}$, 294.9863).

Compounds 3-7 were prepared through the following palladium catalyzed amination reaction, ${ }^{3}$ and the typical yield for is $30 \%$.

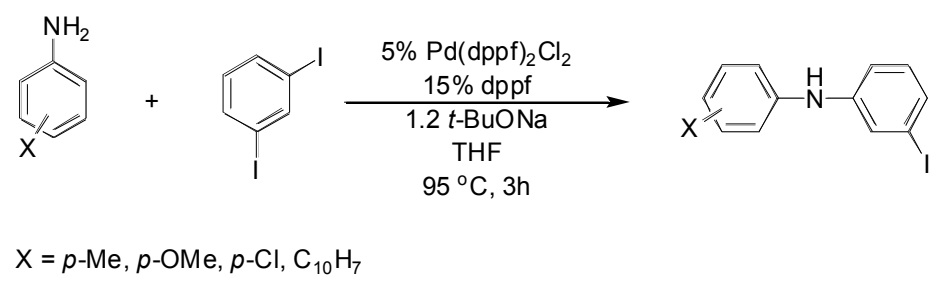

\section{3-Iodo- $N$-p-tolylbenzenamine (4)}

${ }^{1} \mathrm{H}$ NMR $\left(\mathrm{CDCl}_{3}\right) \delta 2.34(\mathrm{~s}, 3 \mathrm{H}), 5.58(\mathrm{~s}, 1 \mathrm{H}), 6.93-7.26(\mathrm{~m}, 7 \mathrm{H}), 7.34(\mathrm{~s}, 1 \mathrm{H}) ;{ }^{13} \mathrm{C} \mathrm{NMR}$ $\left(\mathrm{CDCl}_{3}\right)$ 21.05, 95.26, 115.67, 120.21, 124.93, 129.04, 130.28, 131.00, 132.33, 139.30, 145.87; IR $\left(\mathrm{CDCl}_{3}\right) 3427,3028,2922,1587 \mathrm{~cm}^{-1}$; HRMS m/z 309.0018 (calcd for $\left.\mathrm{C}_{13} \mathrm{H}_{9} \mathrm{IN}, 309.0015\right)$.

\section{3-Iodo- $N$-(4-methoxyphenyl)benzenamine (5)}

${ }^{1} \mathrm{H} \mathrm{NMR}\left(\mathrm{CDCl}_{3}\right) \delta 3.83(\mathrm{~s}, 3 \mathrm{H}), 5.51(\mathrm{~s}, 1 \mathrm{H}), 6.82-7.23(\mathrm{~m}, 8 \mathrm{H}) ;{ }^{13} \mathrm{C} \mathrm{NMR}\left(\mathrm{CDCl}_{3}\right)$ $55.89,95.49,114.66,115.09,123.52,123.87,128.34,131.07,134.73,147.11,156.14$; IR $\left(\mathrm{CDCl}_{3}\right) 3425,3006,2957,1245 \mathrm{~cm}^{-1}$; HRMS m/z 324.9967 (calcd for $\mathrm{C}_{13} \mathrm{H}_{9} \mathrm{INO}$, 324.9964).

\section{3-Iodo- $N$-p-chlorobenzenamine (6)}

${ }^{1} \mathrm{H}$ NMR $\left(\mathrm{CDCl}_{3}\right) \delta 5.64(\mathrm{~s}, 1 \mathrm{H}), 6.96-7.00(\mathrm{~m}, 4 \mathrm{H}), 7.23-7.28(\mathrm{~m}, 3 \mathrm{H}), 7.36(\mathrm{~s}, 1 \mathrm{H}) ;{ }^{13} \mathrm{C}$ $\operatorname{NMR}\left(\mathrm{CDCl}_{3}\right) 95.31,116.79,120.10,126.14,126.83,129.73,130.22,131.17,140.90$, 
144.55; IR $\left(\mathrm{CDCl}_{3}\right) 3427,3061,3034,1583 \mathrm{~cm}^{-1}$; HRMS m/z 328.9473 (calcd for $\left.\mathrm{C}_{12} \mathrm{H}_{9} \mathrm{ClIN}, 328.9468\right)$.

$N$-(3-Iodophenyl)naphthalen-1-amine (7)

${ }^{1} \mathrm{H} \mathrm{NMR}\left(\mathrm{CDCl}_{3}\right) \delta 5.83(\mathrm{~s}, 1 \mathrm{H}), 6.85-6.97(\mathrm{~m}, 2 \mathrm{H}), 7.21(\mathrm{dt}, J=7.6 \mathrm{~Hz}, J=1.3 \mathrm{~Hz}, 1 \mathrm{H})$, $7.30(\mathrm{t}, J=1.9 \mathrm{~Hz}, 1 \mathrm{H}), 7.37-7.56(\mathrm{~m}, 4 \mathrm{H}), 7.65(\mathrm{~d}, J=7.8 \mathrm{~Hz}, 1 \mathrm{H}), 7.88-8.00(\mathrm{~m}, 2 \mathrm{H})$;

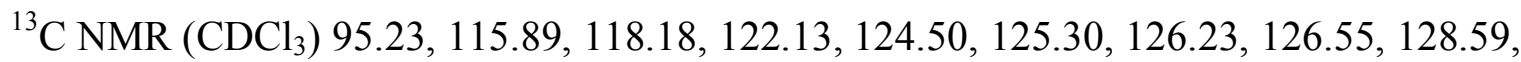
128.84, 129.12, 131.02, 134.94, 137.73, 146.87; IR $\left(\mathrm{CDCl}_{3}\right) 3415,3060,1574 \mathrm{~cm}^{-1}$;

HRMS m/z 345.0018 (calcd for $\mathrm{C}_{18} \mathrm{H}_{12} \mathrm{IN}, 345.0015$ ).

\section{$N$-Allyl-3-iodoaniline (24)}

This compound was prepared according to reported literature. ${ }^{4}{ }^{1} \mathrm{H}$ NMR $\left(\mathrm{CDCl}_{3}\right) \delta 3.74$ (d, $J=5.1 \mathrm{~Hz}, 2 \mathrm{H}), 3.81(\mathrm{~s}, 1 \mathrm{H}), 5.19-5.33(\mathrm{~m}, 2 \mathrm{H}), 5.87-5.99(\mathrm{~m}, 1 \mathrm{H}), 6.57(\mathrm{dd}, J=8.1$ $\mathrm{Hz}, J=2.4 \mathrm{~Hz}, 1 \mathrm{H}), 6.89$ (t, $J=8.1 \mathrm{~Hz}, 1 \mathrm{H}), 6.97$ (t, $J=1.6 \mathrm{~Hz}, 1 \mathrm{H}), 7.05(\mathrm{~d}, J=7.8 \mathrm{~Hz}$, $1 \mathrm{H}) ;{ }^{13} \mathrm{C} \mathrm{NMR}\left(\mathrm{CDCl}_{3}\right) 46.47,95.56,112.54,116.85,121.72,126.55,130.93,135.00$, 149.48; IR $\left(\mathrm{CDCl}_{3}\right) 3417,3076,2847,1590 \mathrm{~cm}^{-1}$; HRMS m/z 258.9862 (calcd for $\left.\mathrm{C}_{9} \mathrm{H}_{10} \mathrm{NI}, 258.9858\right)$.

Representative procedure for the palladium-catalyzed migration reactions. Aryl halide $(0.25 \mathrm{mmol})$, alkyne $(0.25 \mathrm{mmol}), \mathrm{Pd}(\mathrm{OAc})_{2}(2.8 \mathrm{mg}, 0.0125 \mathrm{mmol})$, bis(diphenylphosphino)methane (dppm) $(4.8 \mathrm{mg}, 0.0125 \mathrm{mmol})$ and $\mathrm{CsO}_{2} \mathrm{CCMe}_{3}(\mathrm{CsPiv})$ $(0.117 \mathrm{~g}, 0.5 \mathrm{mmol})$ in $4 \mathrm{~mL}$ of DMF were stirred under $\mathrm{Ar}$ at $100{ }^{\circ} \mathrm{C}$ for $6 \mathrm{~h}$. The reaction mixture was allowed to cool to room temperature, diluted with diethyl ether (50 $\mathrm{mL})$ and washed with $5 \% \mathrm{Na}_{2} \mathrm{CO}_{3}(25 \mathrm{~mL})$. The aqueous layer was re-extracted with diethyl ether $(25 \mathrm{~mL})$. The organic layers were combined, dried $\left(\mathrm{MgSO}_{4}\right)$, filtered, and 
the solvent was removed under reduced pressure. The residue was purified by flash chromatography on silica gel.

For the products reported in entries 1,3, 6, 10,11 of Table 1, GC-mass spectral analysis shows two region-isomers, which can not be separated by flash chromatography, and the ratio of these isomers was determined by ${ }^{1} \mathrm{H}$ NMR spectroscopy. The spectra reported for 8a, 10a, 13a, 16a, 17a, and 25a were taken on obtained mixtures.

\section{(E)-4-(1-Phenylbut-1-enyl)-9H-carbazole (8a)}

${ }^{1} \mathrm{H} \mathrm{NMR}\left(\mathrm{CDCl}_{3}\right) \delta 1.11(\mathrm{t}, J=7.4 \mathrm{~Hz}, 3 \mathrm{H}), 2.94(\mathrm{q}, J=7.4 \mathrm{~Hz}, 2 \mathrm{H}), 6.74(\mathrm{~s}, 1 \mathrm{H}), 7.11-$ $7.20(\mathrm{~m}, 2 \mathrm{H}), 7.35-7.53(\mathrm{~m}, 9 \mathrm{H}), 8.05(\mathrm{~s}, 1 \mathrm{H}), 8.20(\mathrm{~d}, J=7.9 \mathrm{~Hz}, 1 \mathrm{H}) ;{ }^{13} \mathrm{C} \mathrm{NMR}\left(\mathrm{CDCl}_{3}\right)$ $13.37,25.85,109.39,110.71,119.59,120.16,120.93,123.03,123.28,125.66,125.76$, $126.92,128.68,128.90,129.04,138.22,139.90,139.93,140.07,144.57 ; \mathrm{IR}\left(\mathrm{CDCl}_{3}\right)$ 3471, 3056, 2968, 2934, $1599 \mathrm{~cm}^{-1}$; HRMS m/z 297.1522 (calcd for $\mathrm{C}_{22} \mathrm{H}_{19} \mathrm{~N}, 297.1518$ ). (E)-4-(4,4-Dimethylpent-2-en-2-yl)-9H-carbazole (9)

${ }^{1} \mathrm{H} \mathrm{NMR}\left(\mathrm{CDCl}_{3}\right) \delta 1.34(\mathrm{~s}, 9 \mathrm{H}), 2.28(\mathrm{~d}, J=1.3 \mathrm{~Hz}, 3 \mathrm{H}), 5.67(\mathrm{~d}, J=1.4 \mathrm{~Hz}, 1 \mathrm{H}), 6.96$ $(\mathrm{dd}, J=6.9 \mathrm{~Hz}, J=1.2 \mathrm{~Hz}, 1 \mathrm{H}), 7.19-7.42(\mathrm{~m}, 5 \mathrm{H}), 8.06(\mathrm{~s}, 1 \mathrm{H}), 8.14(\mathrm{~d}, J=7.8 \mathrm{~Hz}, 1 \mathrm{H})$; ${ }^{13} \mathrm{C}$ NMR $\left(\mathrm{CDCl}_{3}\right)$ 19.24, 31.20, 33.06, 108.81, 110.58, 119.40, 119.65, 120.21, 122.97, 123.21, 125.57, 125.86, 134.75, 139.55, 139.79, 139.93, 143.33; IR $\left(\mathrm{CDCl}_{3}\right) 3473,2960$, $2867,1600 \mathrm{~cm}^{-1} ;$ HRMS m/z 263.1679 (calcd for $\mathrm{C}_{19} \mathrm{H}_{21} \mathrm{~N}, 263.1674$ ).

\section{(E)-4-(1-Phenylprop-1-enyl)-9H-carbazole (10a)}

${ }^{1} \mathrm{H}$ NMR $\left(\mathrm{CDCl}_{3}\right) \delta 2.47(\mathrm{~s}, 3 \mathrm{H}), 6.78(\mathrm{~s}, 1 \mathrm{H}), 7.12-7.20(\mathrm{~m}, 2 \mathrm{H}), 7.33-7.54(\mathrm{~m}, 9 \mathrm{H}), 8.10$ (s, 1H), $8.14(\mathrm{~d}, J=7.8 \mathrm{~Hz}, 1 \mathrm{H}) ;{ }^{13} \mathrm{C} \mathrm{NMR}\left(\mathrm{CDCl}_{3}\right)$ 19.9, 109.42, 110.68, 119.43, 119.64, $120.23,122.93,123.07,125.79,125.91,126.84,128.59,129.32,129.38,138.30,138.34$, 
139.87, 140.08, 141.37; IR $\left(\mathrm{CDCl}_{3}\right) 3471,3060,3026,1601,1456 \mathrm{~cm}^{-1} ; \mathrm{HRMS} \mathrm{m} / \mathrm{z}$ 283.1367 (calcd for $\mathrm{C}_{18} \mathrm{H}_{19} \mathrm{~N}, 283.1361$ ).

\section{(E)-4-(1,2-Diphenylvinyl)-9H-carbazole (11)}

${ }^{1} \mathrm{H}$ NMR $\left(\mathrm{CDCl}_{3}\right) \delta$ 7.03-7.14 (m, 3H), 7.23-7.42 (m, 14H), $8.03(\mathrm{~s}, 1 \mathrm{H}), 8.37(\mathrm{~d}, J=8.1$ $\mathrm{Hz}, 1 \mathrm{H}) ;{ }^{13} \mathrm{C} \mathrm{NMR}\left(\mathrm{CDCl}_{3}\right)$ 109.75, 110.77, 119.60, 121.31, 121.72, 122.97, 123.39, $125.70,125.93,127.25,127.65,128.49,128.66,129.79,130.21,131.11,137.62,140.03$, 140.27, 140.38, 140.68, 141.26; IR $\left(\mathrm{CDCl}_{3}\right) 3414,3054,1599,1455 \mathrm{~cm}^{-1} ; \mathrm{HRMS} \mathrm{m} / \mathrm{z}$ 345.1522 (calcd for $\mathrm{C}_{26} \mathrm{H}_{19} \mathrm{~N}, 345.1518$ ).

\section{(E)-Ethyl 4-(1-(9H-carbazol-4-yl)but-1-enyl)benzoate (12)}

${ }^{1} \mathrm{H} \mathrm{NMR}\left(\mathrm{CDCl}_{3}\right) \delta 1.90(\mathrm{t}, J=7.5 \mathrm{~Hz}, 3 \mathrm{H}), 1.44(\mathrm{t}, J=7.2 \mathrm{~Hz}, 3 \mathrm{H}), 1.91(\mathrm{q}, J=7.5 \mathrm{~Hz}$, 2H), $4.43(\mathrm{q}, J=7.2 \mathrm{~Hz}, 2 \mathrm{H}), 6.73(\mathrm{~s}, 1 \mathrm{H}), 7.10(\mathrm{dd}, J=6.7 \mathrm{~Hz}, J=1.7 \mathrm{~Hz}, 1 \mathrm{H}), 7.15(\mathrm{td}$, $J=6.8 \mathrm{~Hz}, J=1.5 \mathrm{~Hz}, 1 \mathrm{H}), 7.37-7.46(\mathrm{~m}, 4 \mathrm{H}), 7.53(\mathrm{~d}, J=8.1 \mathrm{~Hz}, 2 \mathrm{H}), 8.10-8.20(\mathrm{~m}$, $4 \mathrm{H}) ;{ }^{13} \mathrm{C} \mathrm{NMR}\left(\mathrm{CDCl}_{3}\right) 13.25,14.64,25.99,61.19,109.61,110.78,119.59,119.93$, $120.76,122.81,123.09,125.64,125.82,128.17,128.77,128.86,129.96,139.43,139.91$, 140.07, 142.75, 146.72, 166.83; IR $\left(\mathrm{CDCl}_{3}\right) 3472,2968,2873,1710 \mathrm{~cm}^{-1}$; HRMS m/z 369.1736 (calcd for $\mathrm{C}_{25} \mathrm{H}_{23} \mathrm{NO}_{2}, 369.1729$ ).

(E)-4-(1-(2-Methoxyphenyl)but-1-enyl)-9H-carbazole (13a)

${ }^{1} \mathrm{H} \mathrm{NMR}\left(\mathrm{CDCl}_{3}\right) \delta 1.00(\mathrm{t}, J=7.5 \mathrm{~Hz}, 3 \mathrm{H}), 2.80(\mathrm{q}, J=7.5 \mathrm{~Hz}, 2 \mathrm{H}), 3.84(\mathrm{~s}, 3 \mathrm{H}), 6.77$ (s, 1H), $6.95(\mathrm{~d}, J=8.0 \mathrm{~Hz}, 1 \mathrm{H}), 6.97-7.17(\mathrm{~m}, 3 \mathrm{H}), 7.29-7.53(\mathrm{~m}, 6 \mathrm{H}), 8.11(\mathrm{~s}, 1 \mathrm{H}), 8.36$ $(\mathrm{d}, J=7.8 \mathrm{~Hz}, 1 \mathrm{H}) ;{ }^{13} \mathrm{C} \mathrm{NMR}\left(\mathrm{CDCl}_{3}\right) 13.28,26.05,55.53,109.12,110.46,110.80$, $118.27,119.31,120.18,120.42,121.18,123.51,124.74,125.51,125.60,127.26,128.32$, 130.06, 139.87, 140.08, 144.02, 157.78; IR $\left(\mathrm{CDCl}_{3}\right) 3472,2966,2934,1245 \mathrm{~cm}^{-1}$; HRMS m/z 327.1628 (calcd for $\mathrm{C}_{23} \mathrm{H}_{21} \mathrm{NO}, 327.1623$ ). 


\section{(E)-5-(1,2-Diphenylvinyl)-3-methyl-9H-carbazole (14)}

${ }^{1} \mathrm{H} \mathrm{NMR}\left(\mathrm{CDCl}_{3}\right) \delta 2.36(\mathrm{~s}, 3 \mathrm{H}), 7.02-7.05(\mathrm{~m}, 2 \mathrm{H}), 7.20-7.40(\mathrm{~m}, 14 \mathrm{H}), 7.95(\mathrm{~s}, 1 \mathrm{H})$, $8.10(\mathrm{~s}, 1 \mathrm{H}) ;{ }^{13} \mathrm{C} \mathrm{NMR}\left(\mathrm{CDCl}_{3}\right) 21.84,109.76,110.36,121.26,121.58,123.11,123.51$, $125.51,127.16,127.19,127.59,128.47,128.54,128.64,129.69,130.32,131.08,137.77$, 138.20, 140.18, 140.53, 140.66, 141.40; IR $\left(\mathrm{CDCl}_{3}\right) 3413,3053,3022,1599,1491 \mathrm{~cm}^{-1}$; HRMS m/z 359.1678 (calcd for $\mathrm{C}_{27} \mathrm{H}_{21} \mathrm{~N}, 359.1674$ ).

\section{(E)-5-(1,2-Diphenylvinyl)-3-methoxy-9H-carbazole (15)}

${ }^{1} \mathrm{H}$ NMR $\left(\mathrm{CDCl}_{3}\right) \delta 3.58(\mathrm{~s}, 3 \mathrm{H}), 7.02-7.07(\mathrm{~m}, 3 \mathrm{H}), 7.22-7.42(\mathrm{~m}, 13 \mathrm{H}), 7.81(\mathrm{~d}, J=2.5$ $\mathrm{Hz}, 1 \mathrm{H}), 7.98(\mathrm{~s}, 1 \mathrm{H}) ;{ }^{13} \mathrm{C} \mathrm{NMR}\left(\mathrm{CDCl}_{3}\right) 55.85,105.21,110.09,111.49,115.78,121.45$, $121.55,123.59,125.59,127.29,127.75,128.67,129.72,130.33,131.32,134.83,137.67$, 140.10, 140.53, 141.08, 141.13, 153.50; IR $\left(\mathrm{CDCl}_{3}\right) 3415,3054,2949,1582,1478 \mathrm{~cm}^{-1}$; HRMS m/z 375.1629 (calcd for $\mathrm{C}_{27} \mathrm{H}_{21} \mathrm{NO}, 375.1623$ ).

\section{(E)-3-Chloro-5-(1-phenylbut-1-enyl)-9H-carbazole (16a)}

${ }^{1} \mathrm{H} \mathrm{NMR}\left(\mathrm{CDCl}_{3}\right) \delta 1.09(\mathrm{t}, J=7.5 \mathrm{~Hz}, 3 \mathrm{H}), 2.91(\mathrm{q}, J=7.5 \mathrm{~Hz}, 2 \mathrm{H}), 6.73(\mathrm{~s}, 1 \mathrm{H}), 7.14$ $(\mathrm{d}, J=7.2 \mathrm{~Hz}, 1 \mathrm{H}), 7.29-7.52(\mathrm{~m}, 10 \mathrm{H}), 8.08(\mathrm{~s}, 1 \mathrm{H}), 8.19(\mathrm{~d}, J=1.5 \mathrm{~Hz}, 1 \mathrm{H}) ;{ }^{13} \mathrm{C} \mathrm{NMR}$ $\left(\mathrm{CDCl}_{3}\right) 13.35,25.76,109.62,111.66,120.52,122.58,124.46,124.88,125.85,126.38$ $127.07,128.77,129.22,129.72,137.98,138.17,140.04,140.61,143.97 ; \mathrm{IR}\left(\mathrm{CDCl}_{3}\right)$ 3471, 2944, $2833 \mathrm{~cm}^{-1}$; HRMS m/z 331.1132 (calcd for $\mathrm{C}_{22} \mathrm{H}_{18} \mathrm{NCl}, 331.1128$ ).

\section{(E)-7-(1-Phenylbut-1-enyl)-11H-benzo[a]carbazole (17a)}

${ }^{1} \mathrm{H}$ NMR $\left(\mathrm{CDCl}_{3}\right) \delta 1.10(\mathrm{t}, J=7.6 \mathrm{~Hz}, 3 \mathrm{H}), 2.95(\mathrm{q}, J=7.6 \mathrm{~Hz}, 2 \mathrm{H}), 6.75(\mathrm{~s}, 1 \mathrm{H}), 7.16$ $(\mathrm{dd}, J=7.3 \mathrm{~Hz}, J=1.2 \mathrm{~Hz}, 1 \mathrm{H}), 7.26-7.60(\mathrm{~m}, 10 \mathrm{H}), 7.98(\mathrm{~d}, J=7.5 \mathrm{~Hz}, 1 \mathrm{H}), 8.14(\mathrm{~d}, J$ $=8.1 \mathrm{~Hz}, 1 \mathrm{H}), 8.23(\mathrm{~d}, J=8.1 \mathrm{~Hz}, 1 \mathrm{H}), 8.87(\mathrm{~s}, 1 \mathrm{H}) ;{ }^{13} \mathrm{C} \mathrm{NMR}\left(\mathrm{CDCl}_{3}\right)$ 13.33, 26.10, $109.83,118.46,120.14,120.55,120.61,121.11,121.81,121.93,124.67,125.53,125.71$, 
126.90, 128.66, 129.03, 129.06, 129.11, 132.25, 135.29, 138.20, 139.06, 139.29, 144.50;

IR $\left(\mathrm{CDCl}_{3}\right)$ 3472, 3060, 2969, 1572 $\mathrm{cm}^{-1}$; HRMS m/z 347.1682 (calcd for $\mathrm{C}_{26} \mathrm{H}_{21} \mathrm{~N}$, 347.1674).

(E)-3-Methyl-4-(1-phenylbut-1-en-2-yl)-1H-indole (25a)

${ }^{1} \mathrm{H} \mathrm{NMR}\left(\mathrm{CDCl}_{3}\right) \delta 1.09(\mathrm{t}, J=7.2 \mathrm{~Hz}, 3 \mathrm{H}), 2.36(\mathrm{~s}, 3 \mathrm{H}), 2.77(\mathrm{q}, J=7.2 \mathrm{~Hz}, 2 \mathrm{H}), 6.47$

(s, 1H), 6.96-6.99 (m, 2H), $7.18(\mathrm{t}, J=7.8 \mathrm{~Hz}, 1 \mathrm{H}), 7.27-7.32(\mathrm{~m}, 2 \mathrm{H}), 7.41$ (d, $J=4.1$

$\mathrm{Hz}, 4 \mathrm{H}), 7.93(\mathrm{~s}, 1 \mathrm{H}) ;{ }^{13} \mathrm{C} \mathrm{NMR}\left(\mathrm{CDCl}_{3}\right)$ 13.24, 13.39, 27.13, 110.03, 112.46, 119.70,

$121.66,122.86,125.59,126.59,128.53,128.88,129.04,137.34,137.90,138.40,144.39$;

IR $\left(\mathrm{CDCl}_{3}\right) 3418,3021,2964,1598 \mathrm{~cm}^{-1}$; HRMS m/z 261.1518 (calcd for $\mathrm{C}_{19} \mathrm{H}_{19} \mathrm{~N}$, 261.1521).

\section{References}

(1) Campo, M. A.; Larock, R. C. Org. Lett. 2000, 2, 3675.

(2) Liu, Z.; Larock, R. C. Org. Lett. 2003, 5, 4673.

(3) Driver, M. S.; Hartwig, J. F. J. Am. Chem. Soc. 1996, 118, 7217.

(4) Caddick, S.; Kofie, W. Tetrahedron Lett. 2002, 43, 9347. 
STAYDARD 1 H OBSERVE

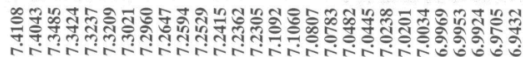
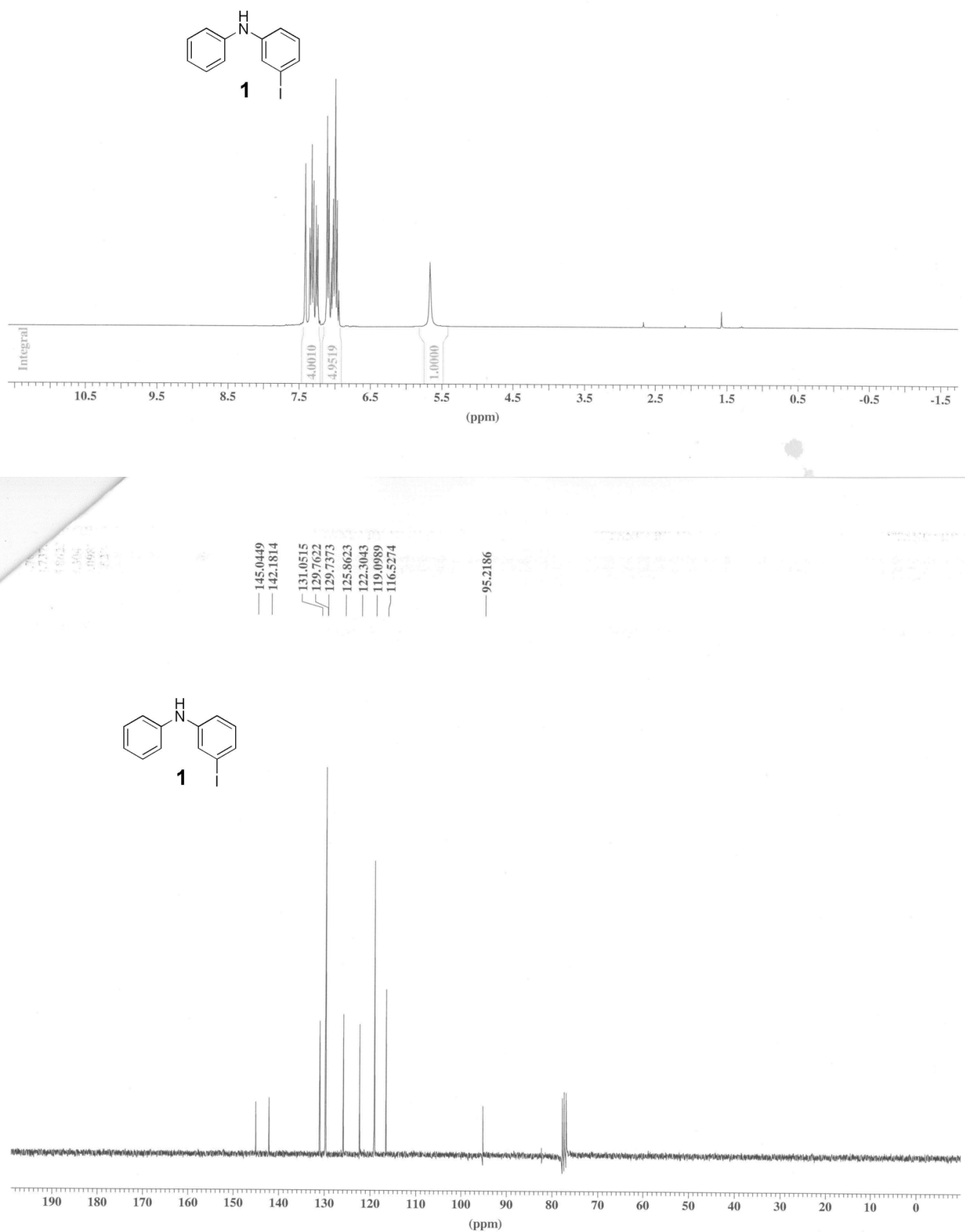

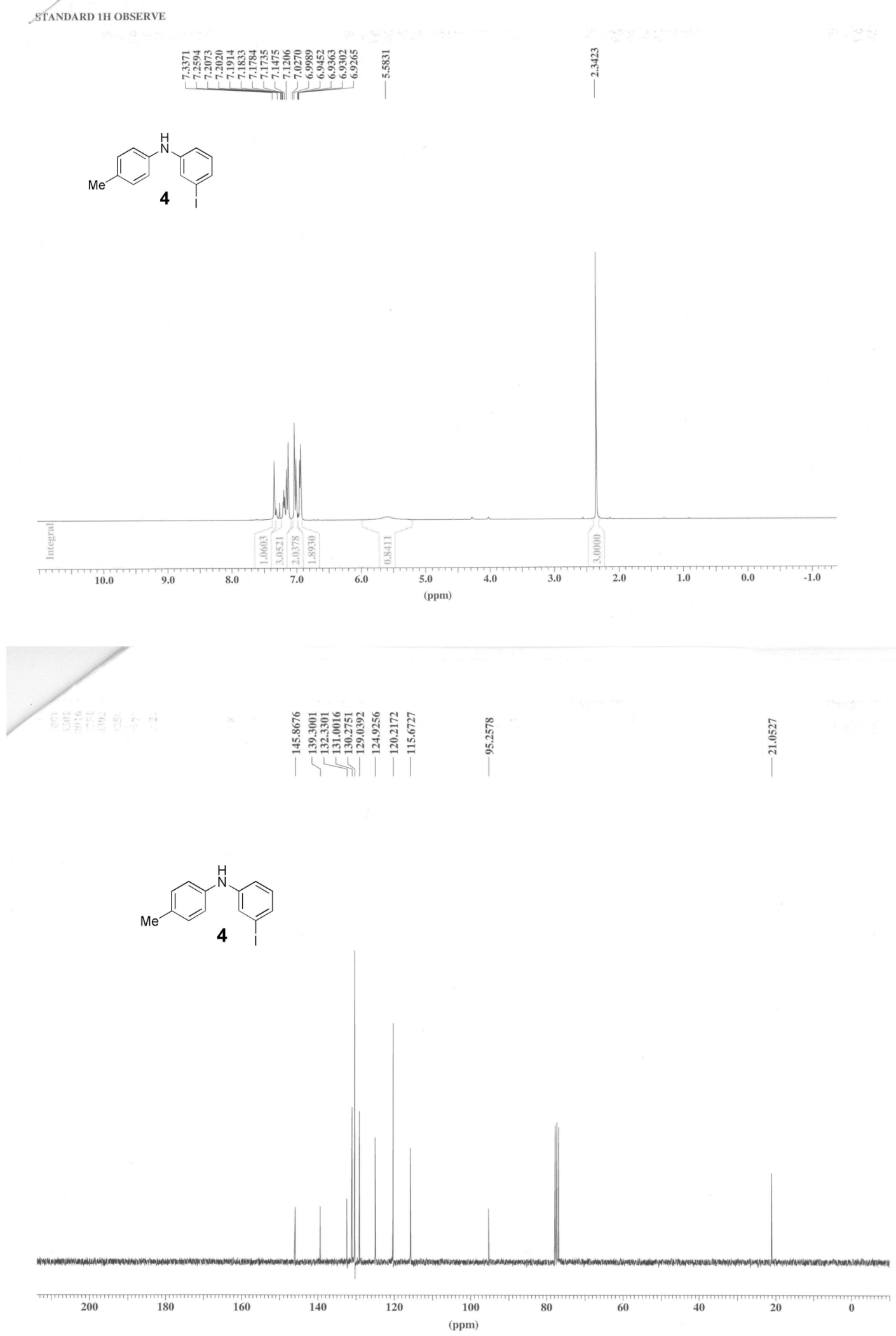
STANDARD 1H OBSERVE
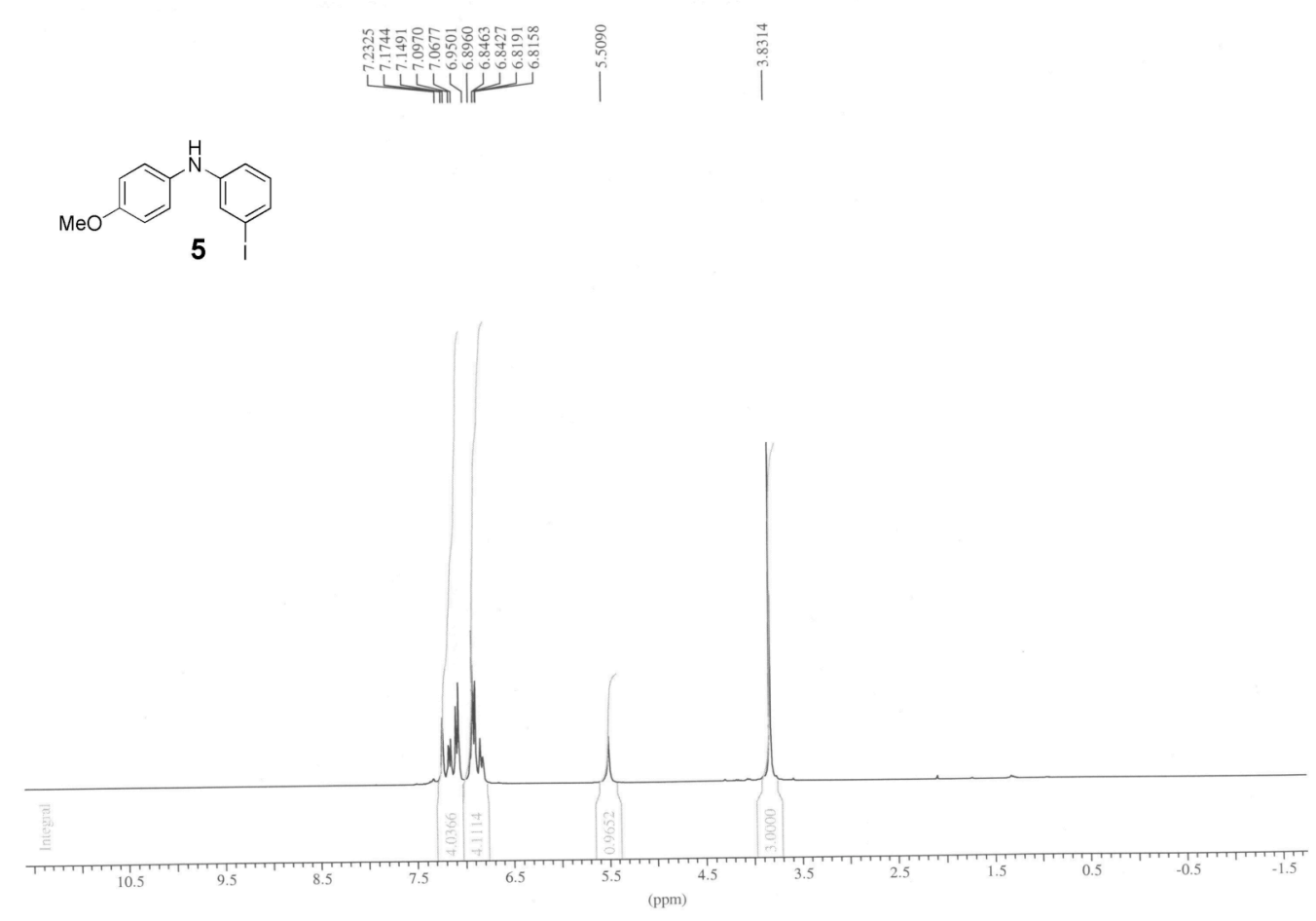

I

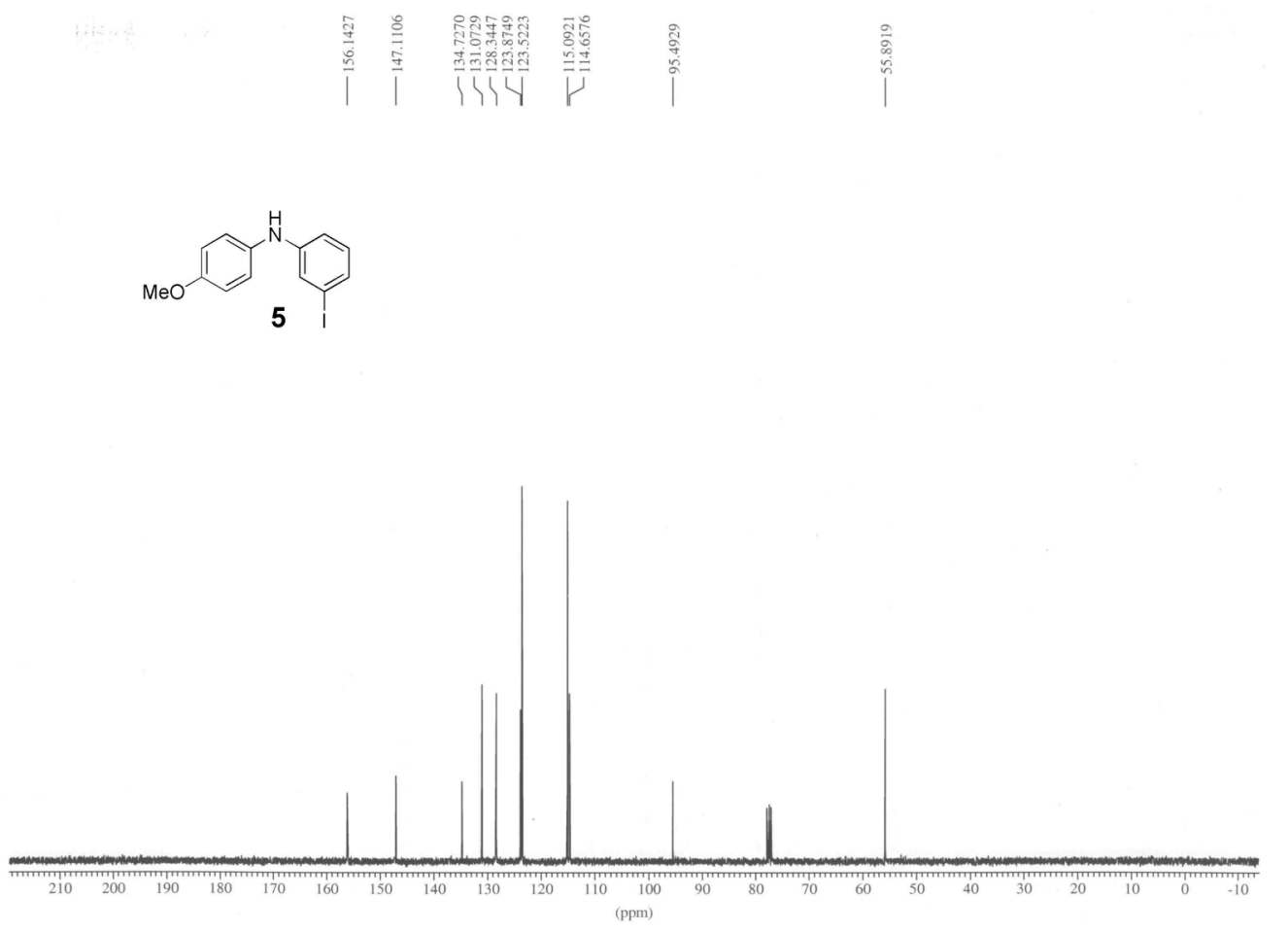


ST ANDARD 1H OBSERVE
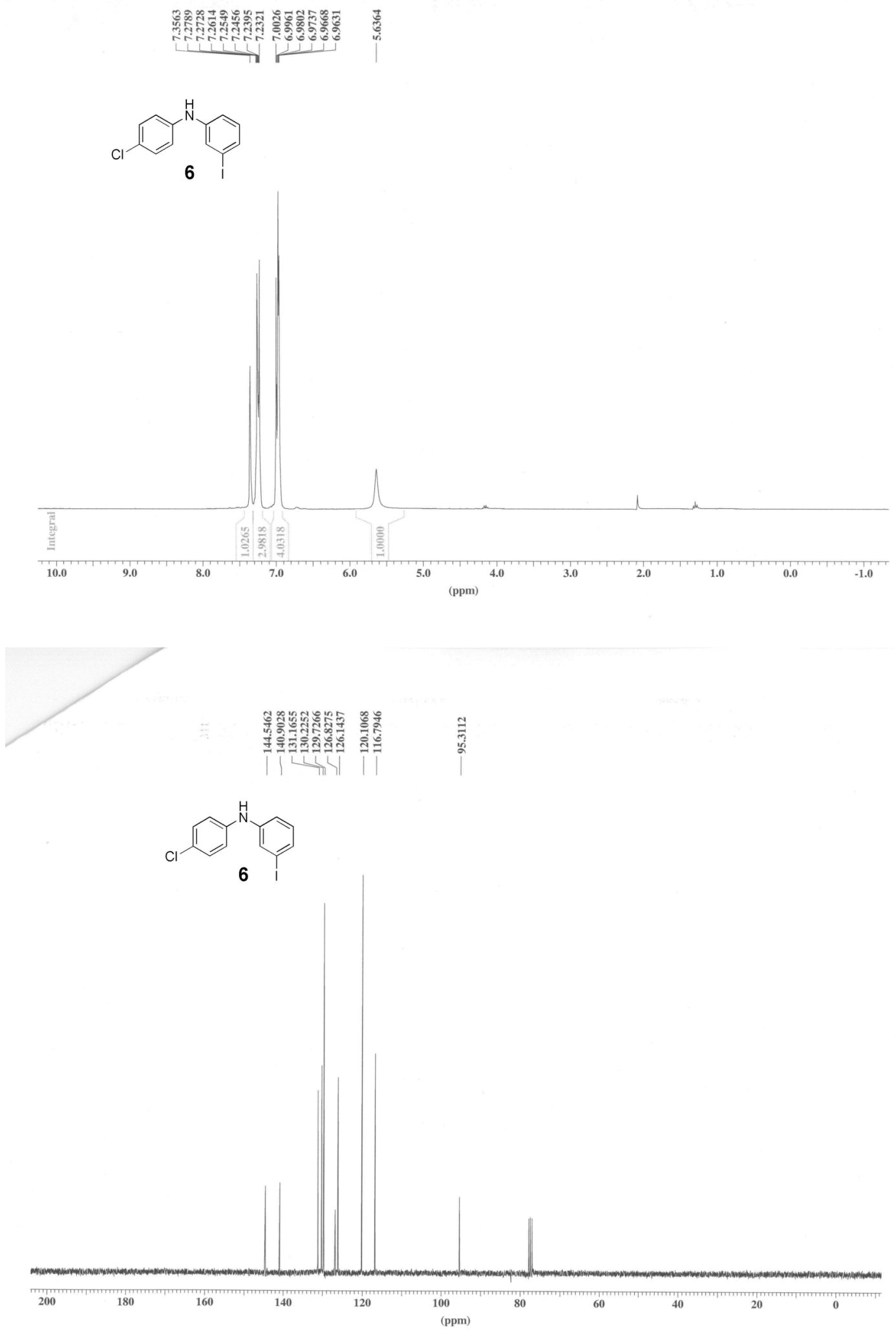

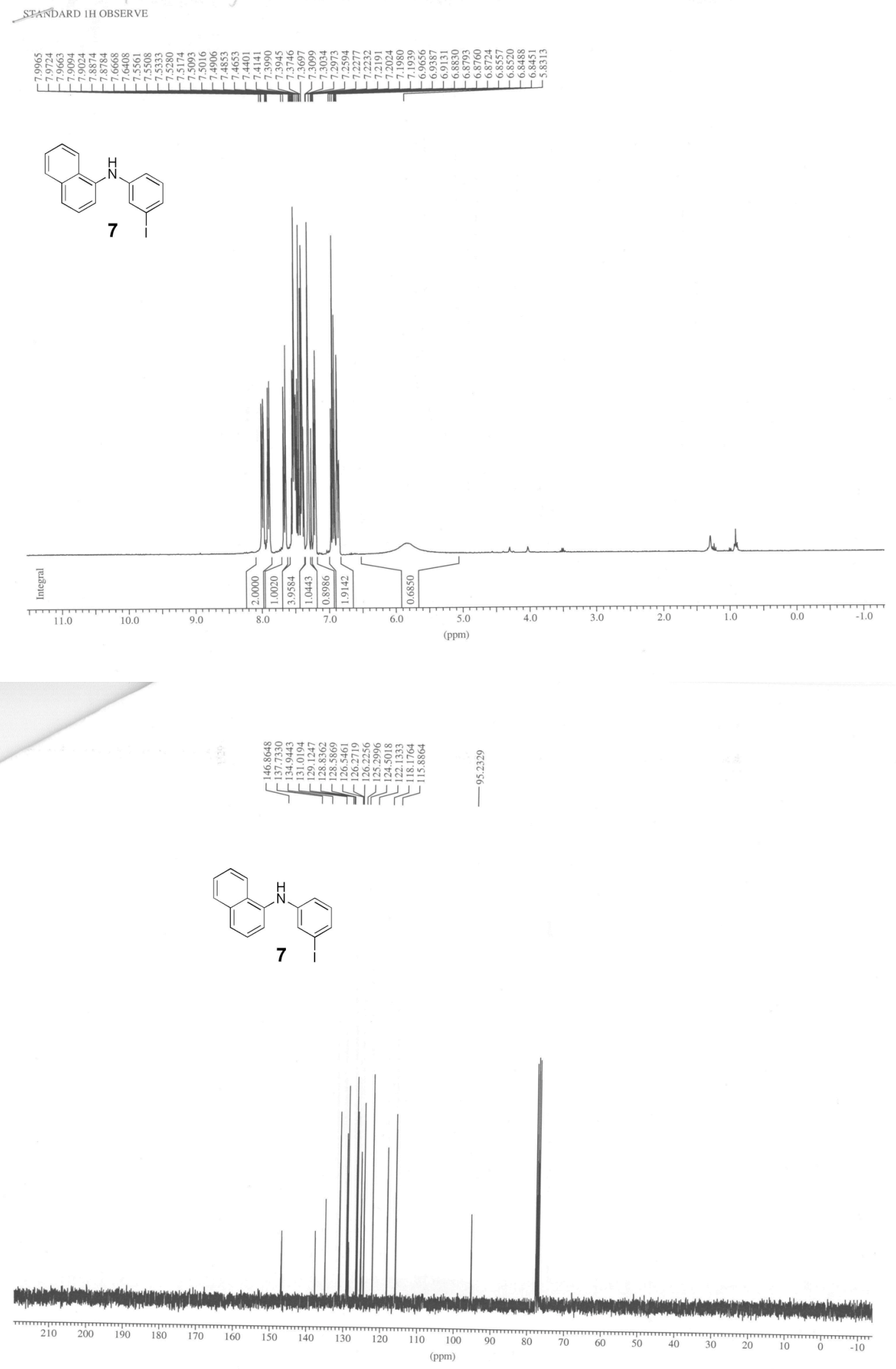

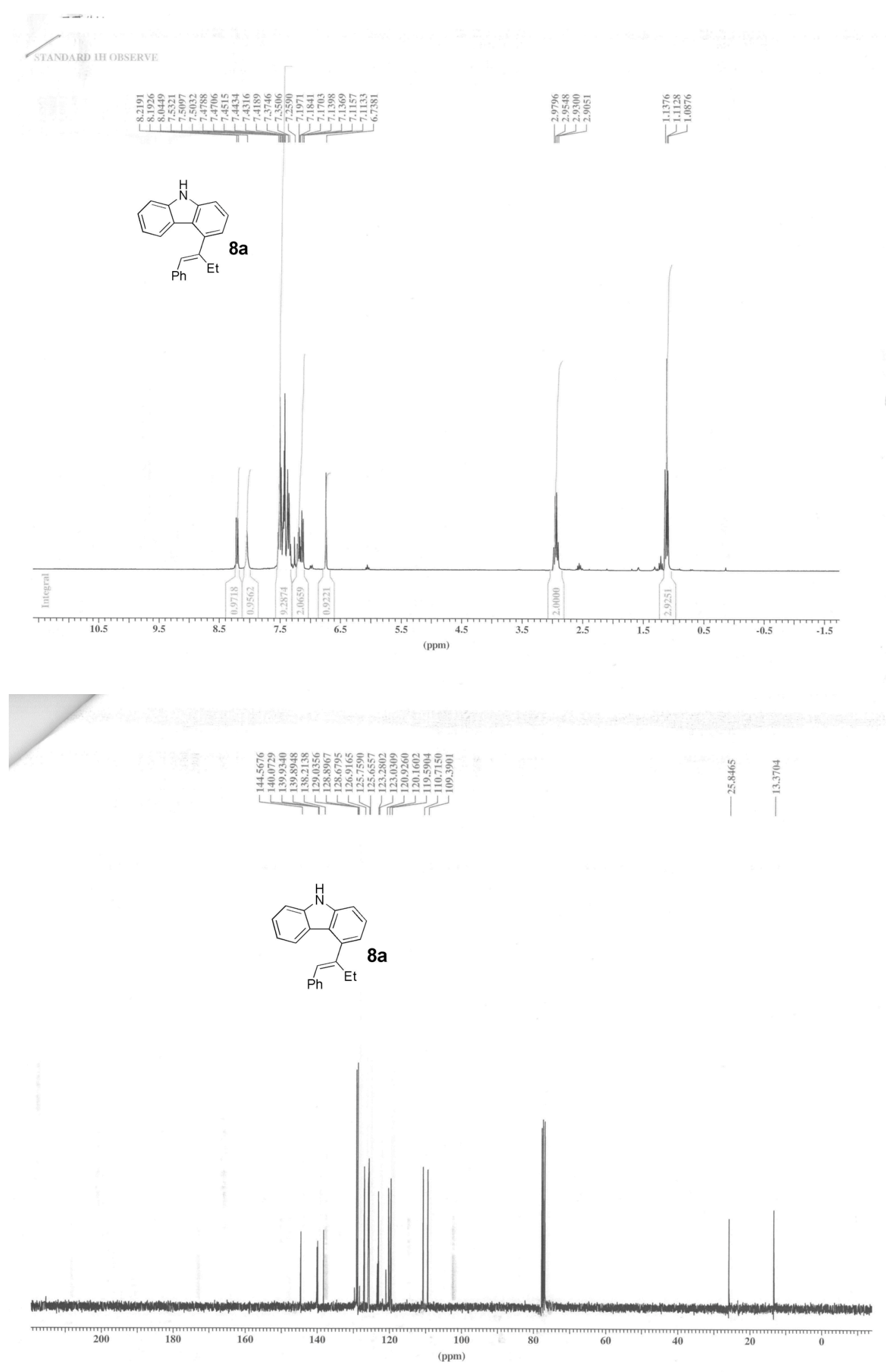
STANDARD 1H OBSERVE
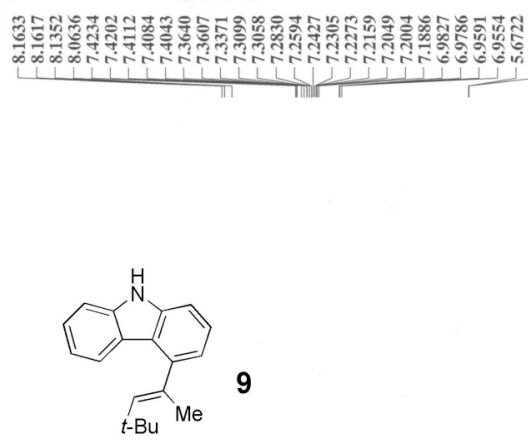

9
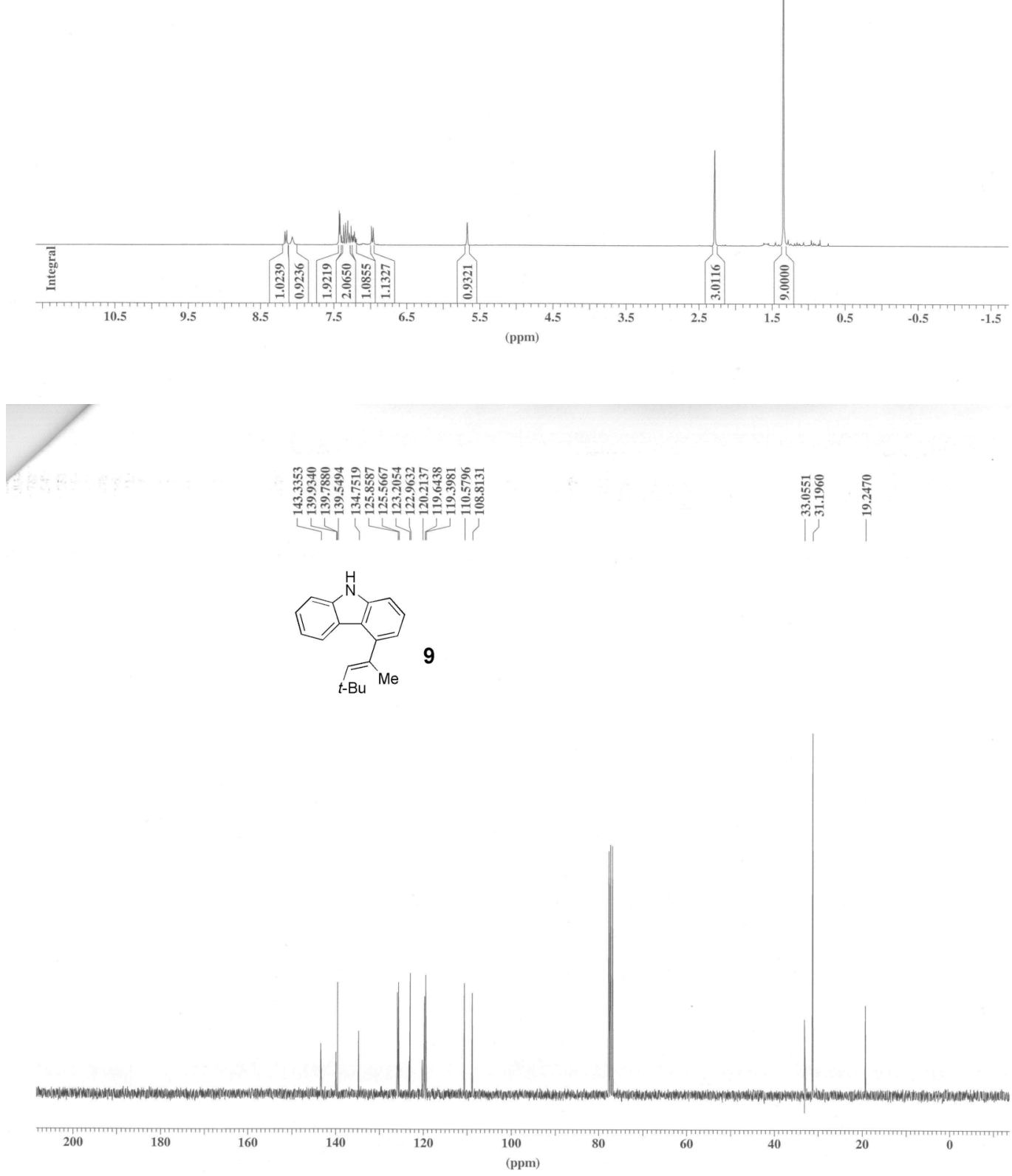
STANDARD IH OBSERVE
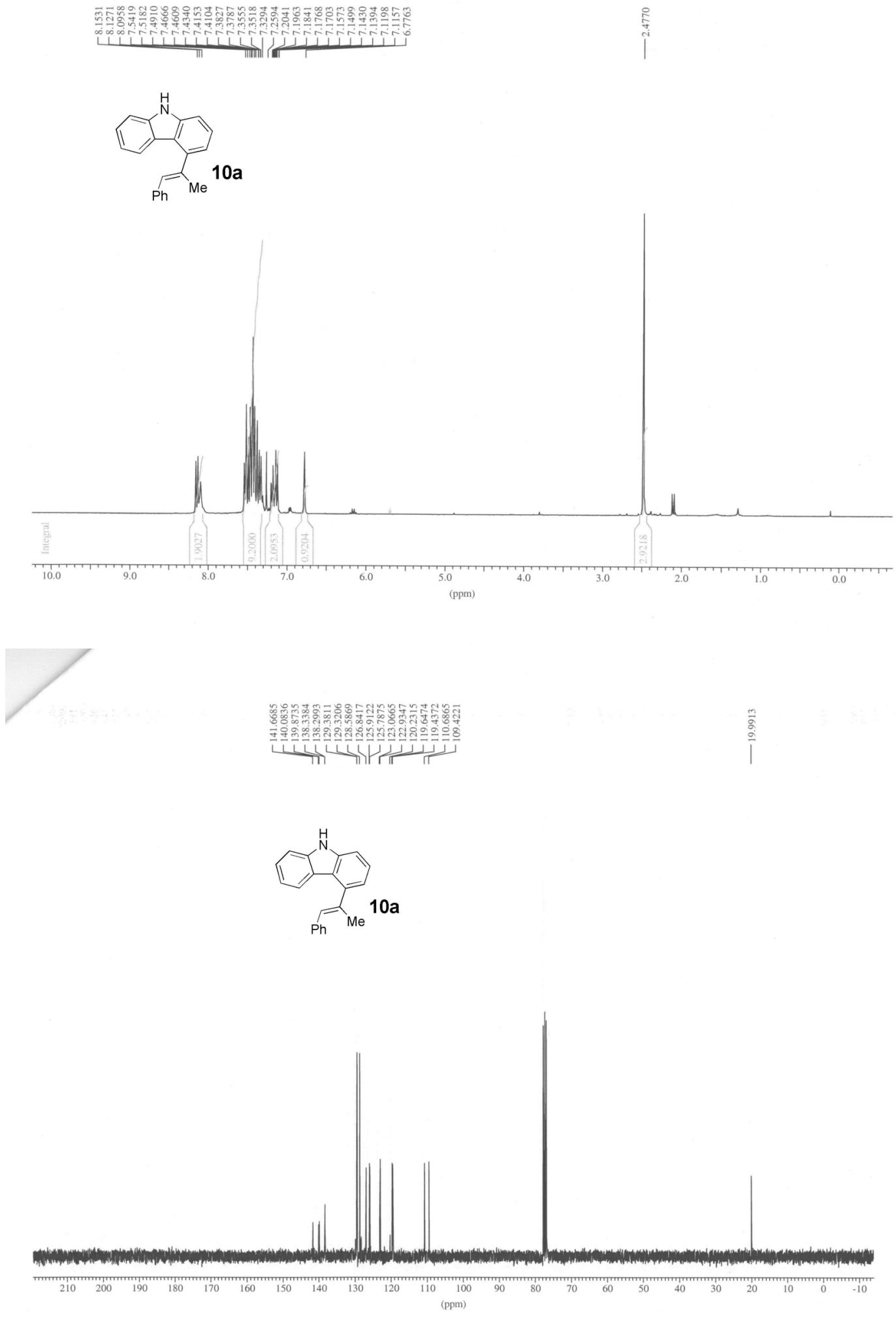
STANDARD 1H OBSERVE
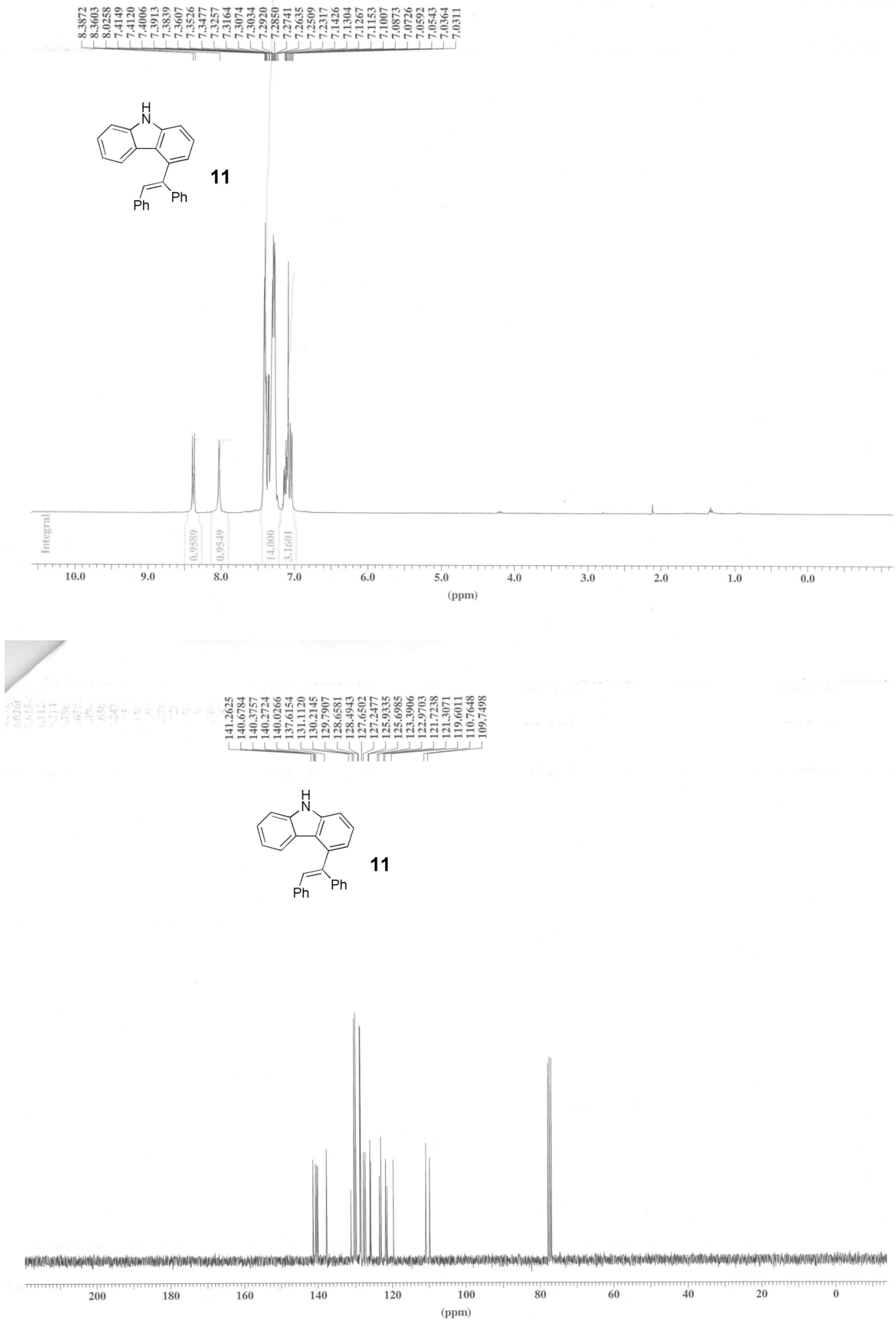


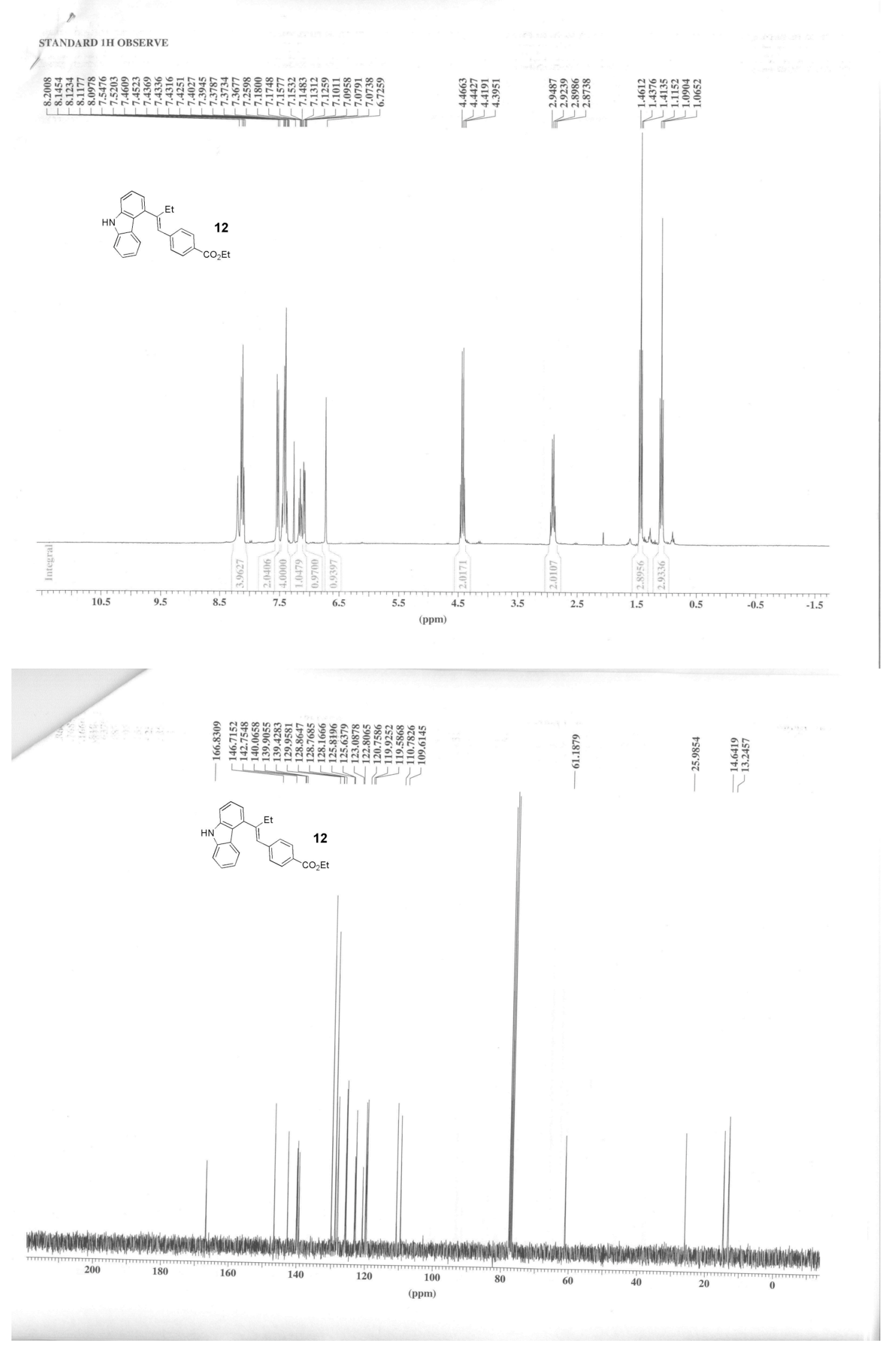



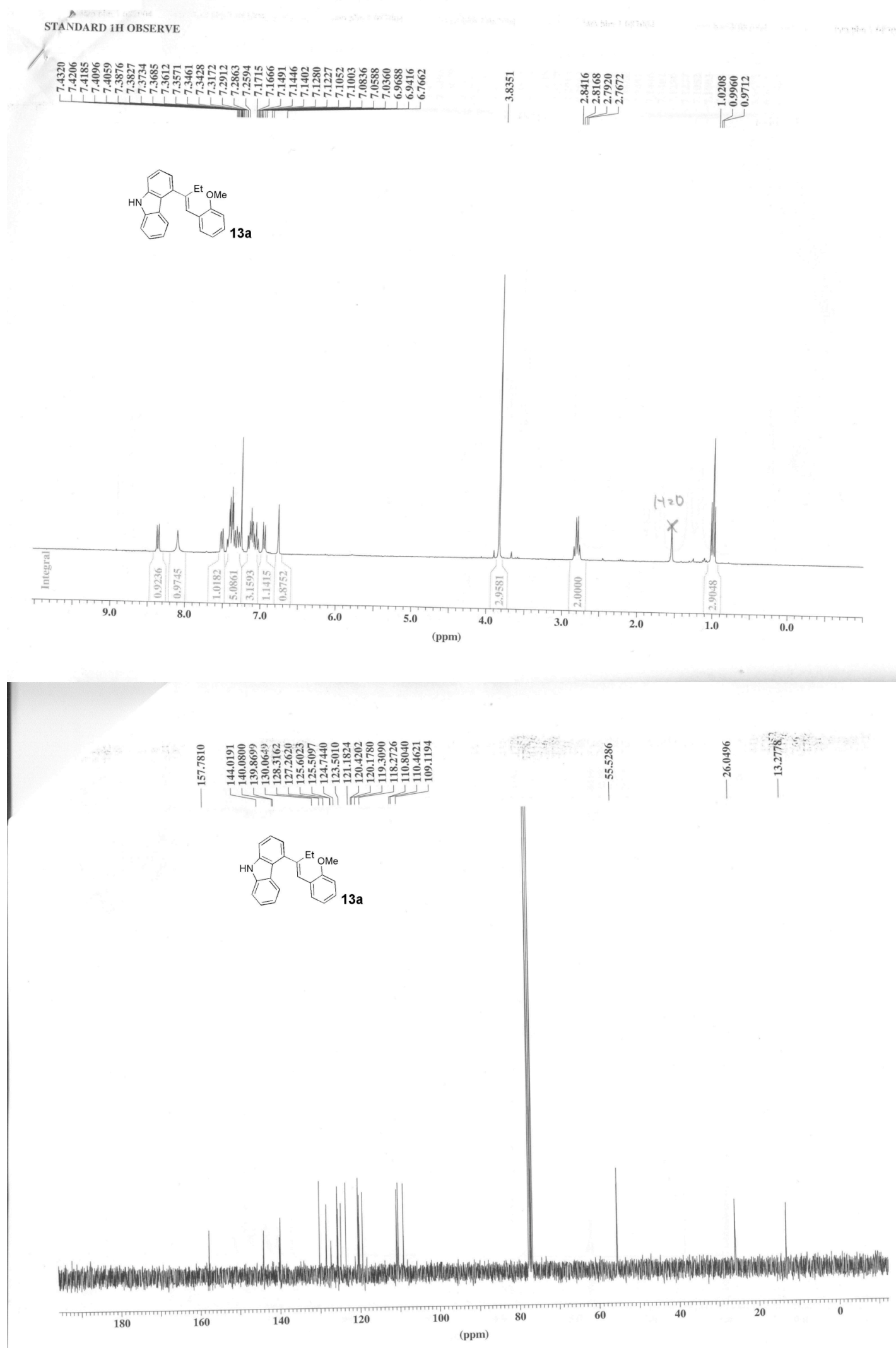
STANDARD 1H OBSERVE

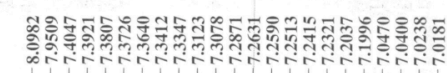

啇
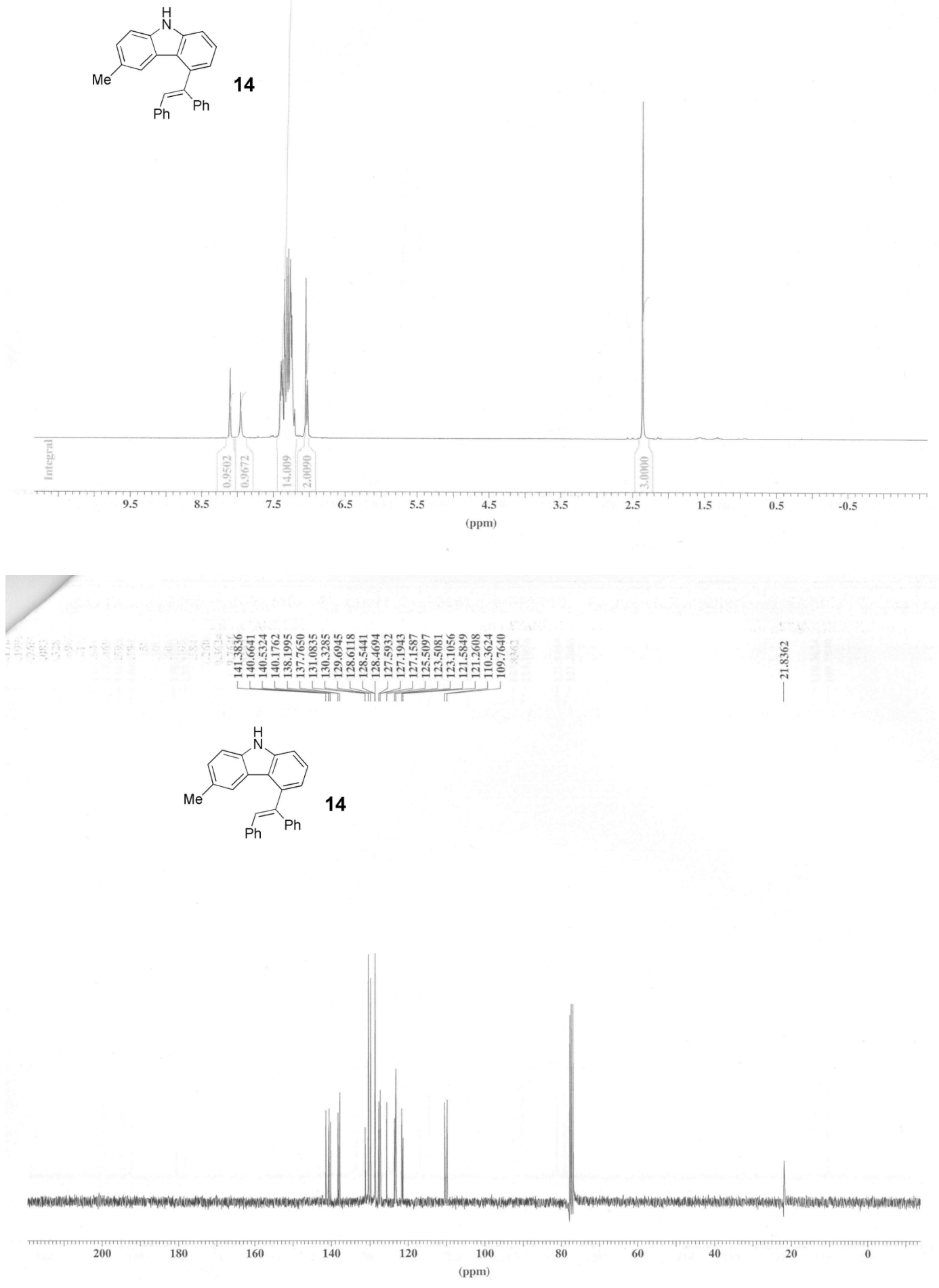
$\int_{\text {STANDARD } 1 H \text { OBSERV }}$
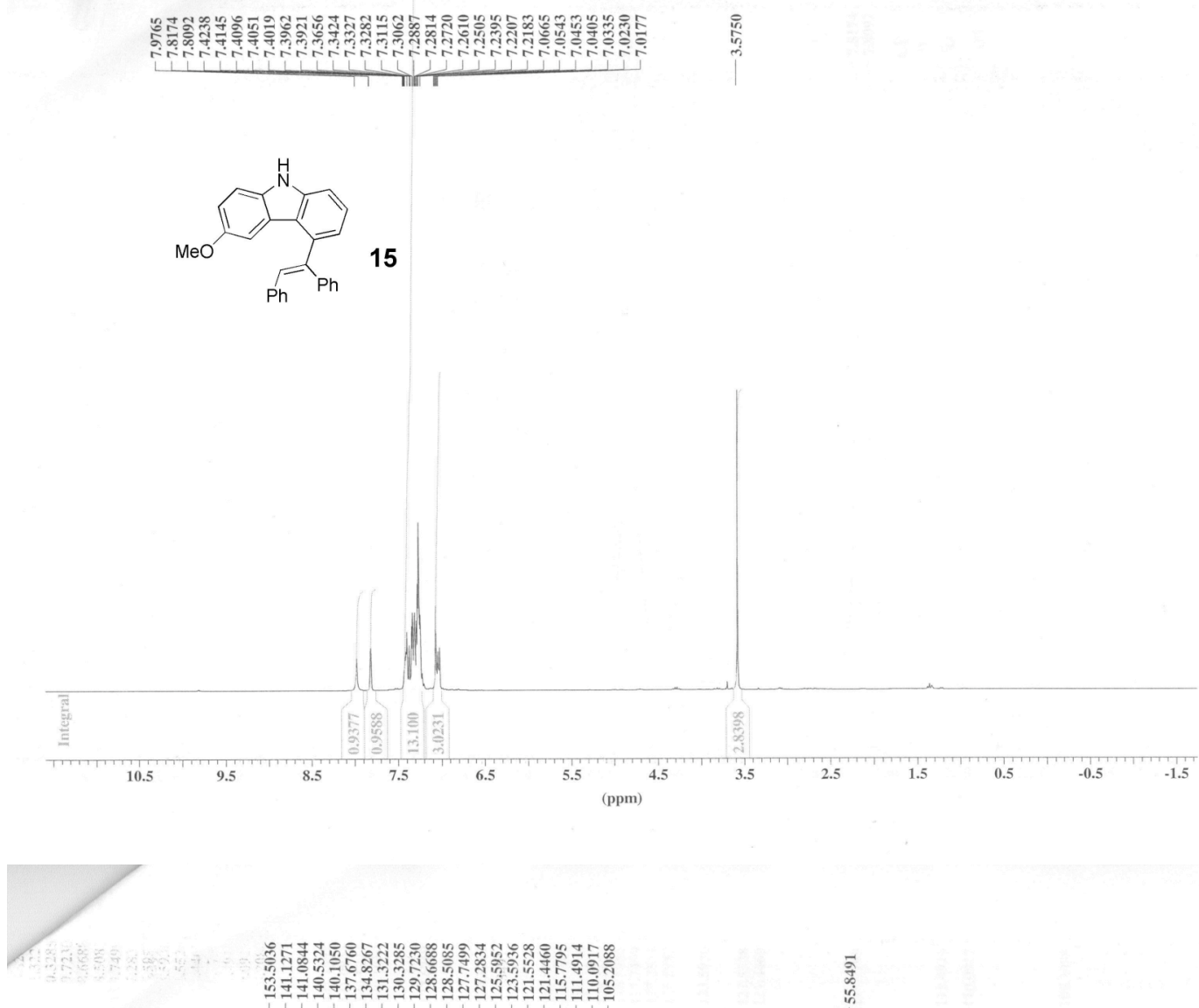

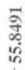

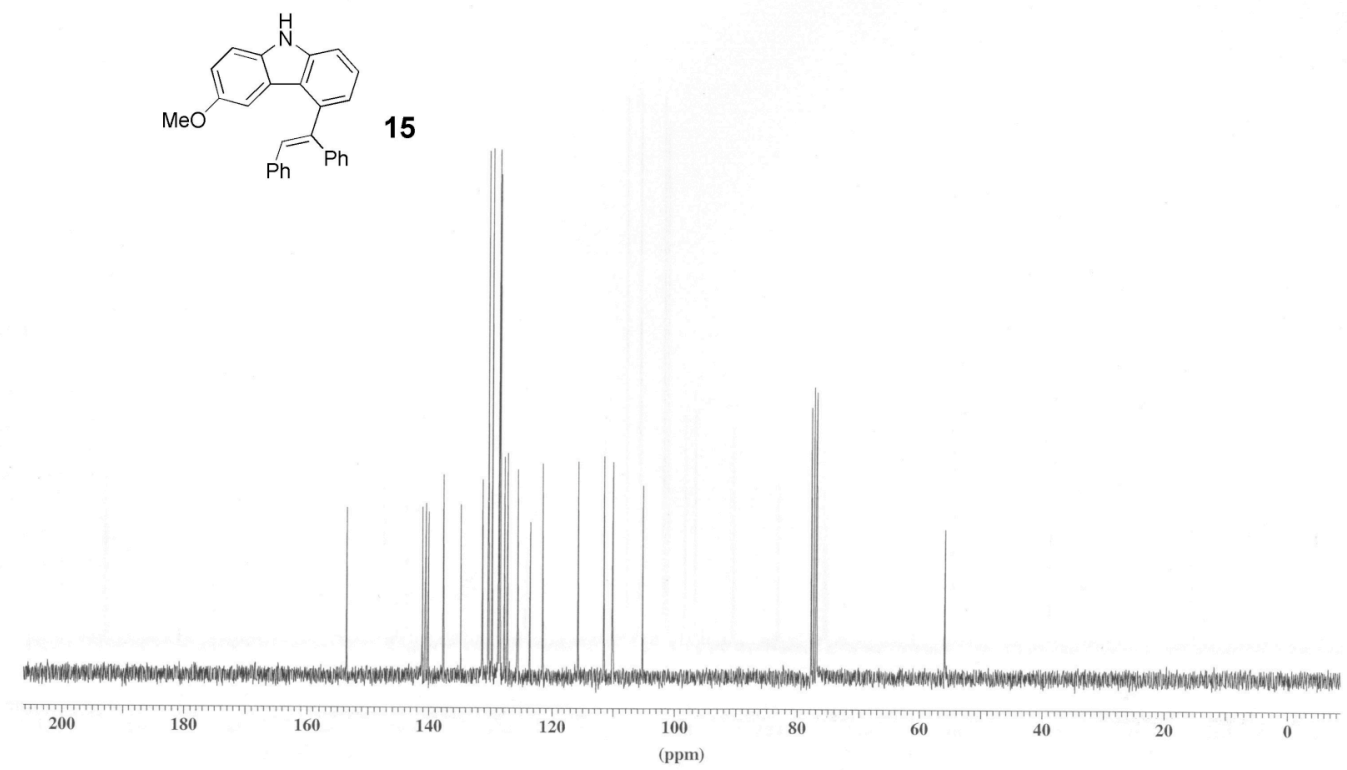



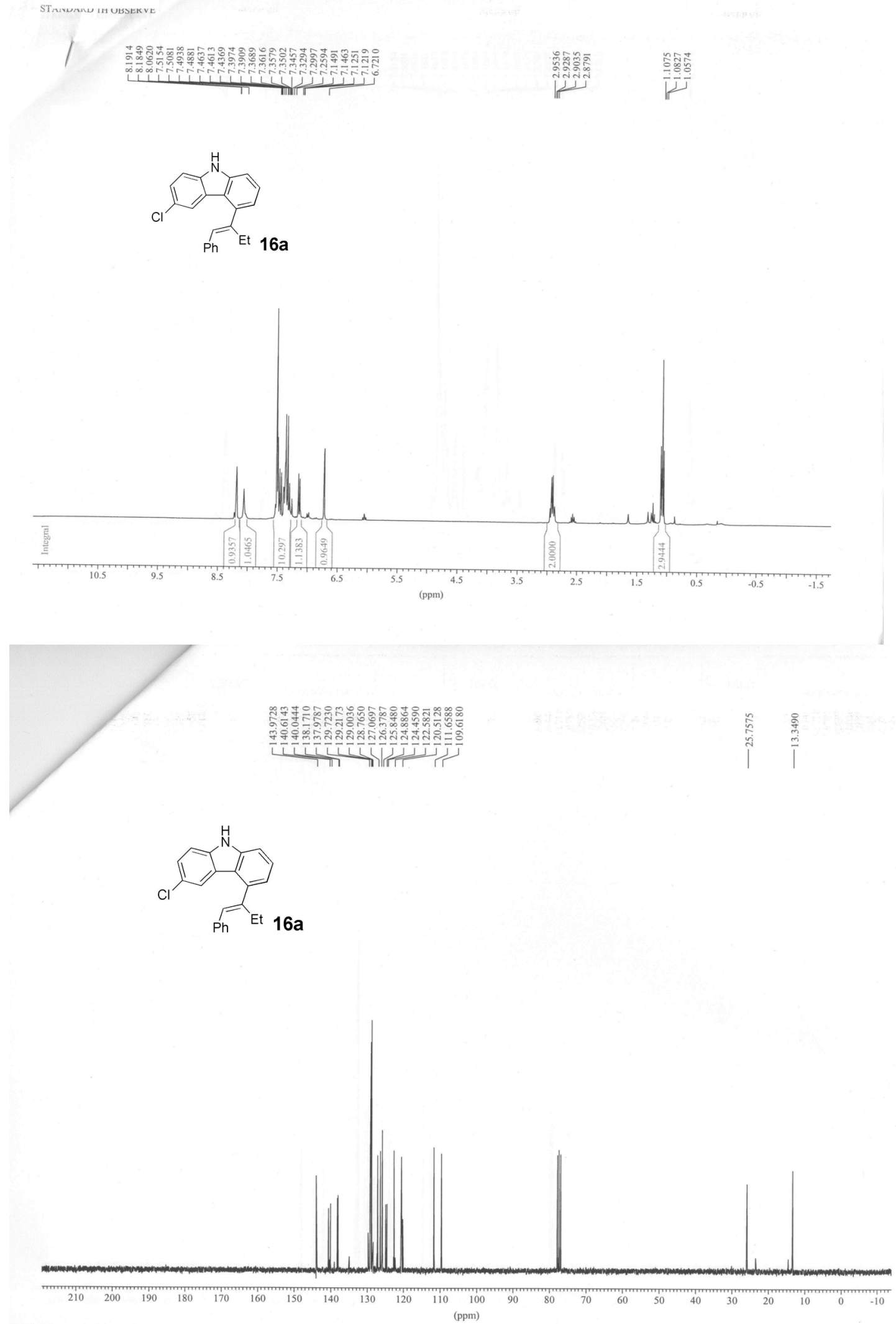

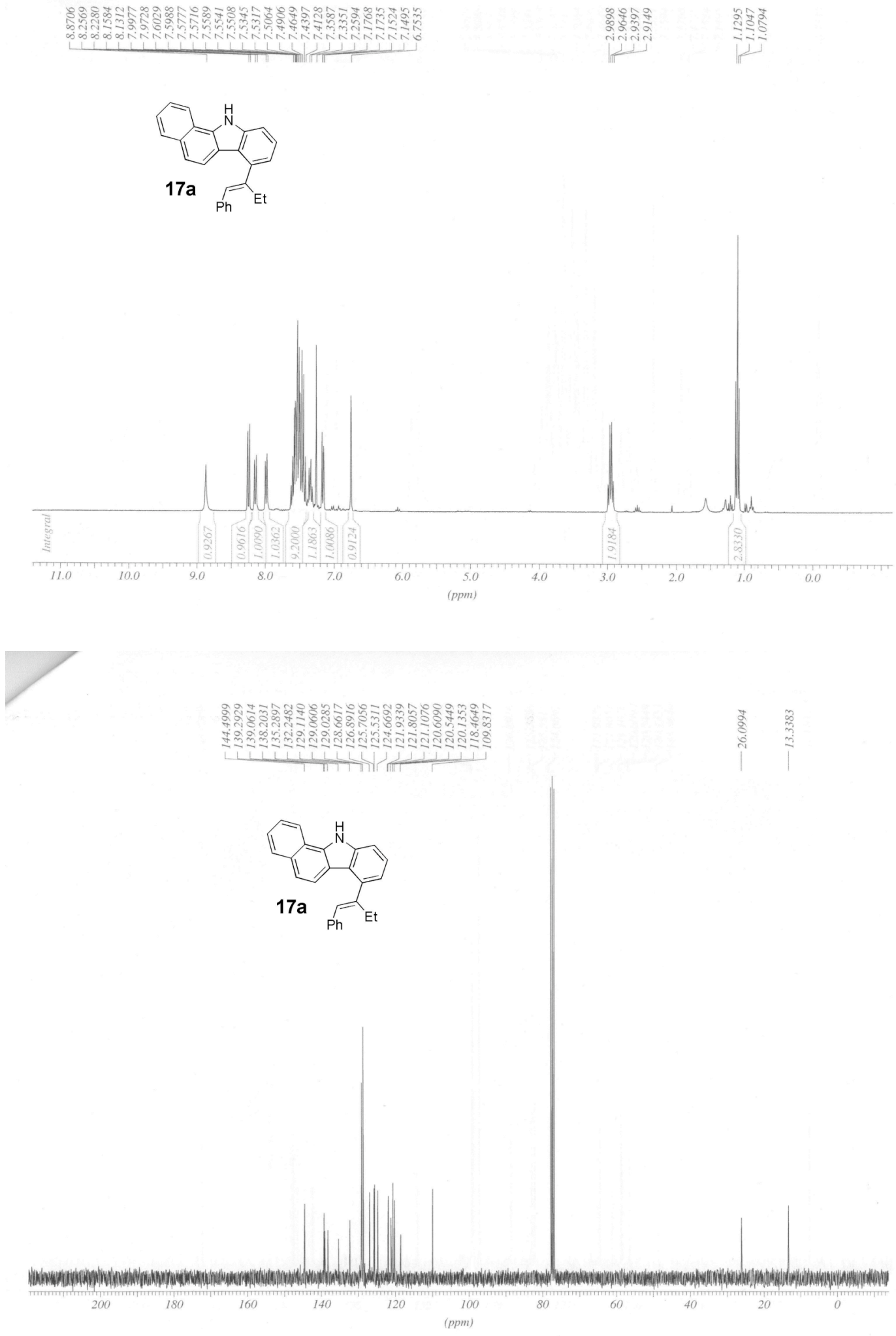


\section{S. ANDARD 1H OBSERVE}
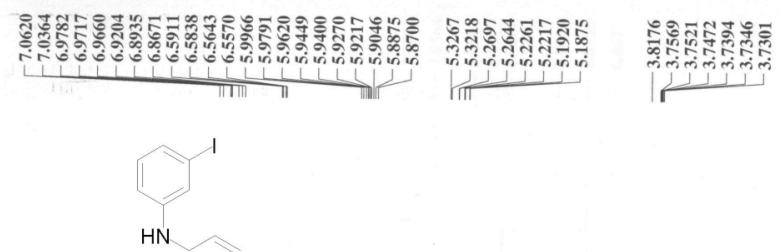

24
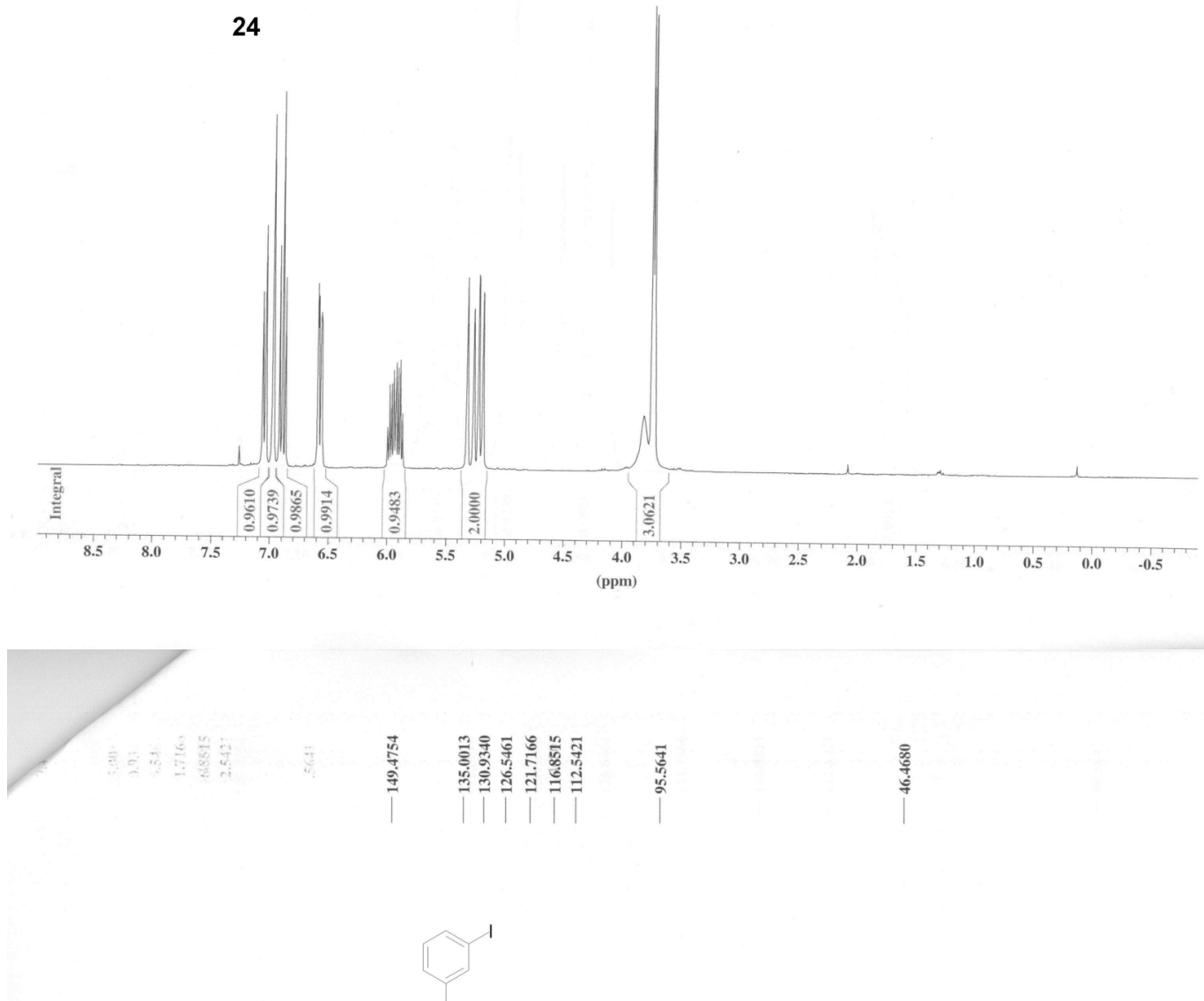

$\mathrm{HN}$

24

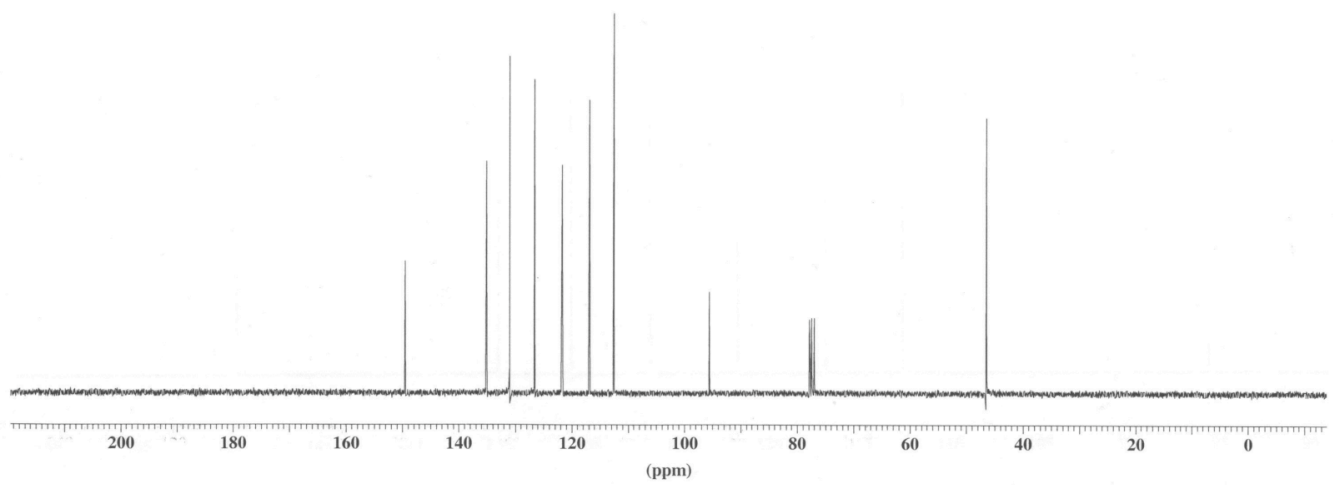


$400 \mathrm{~Hz}$
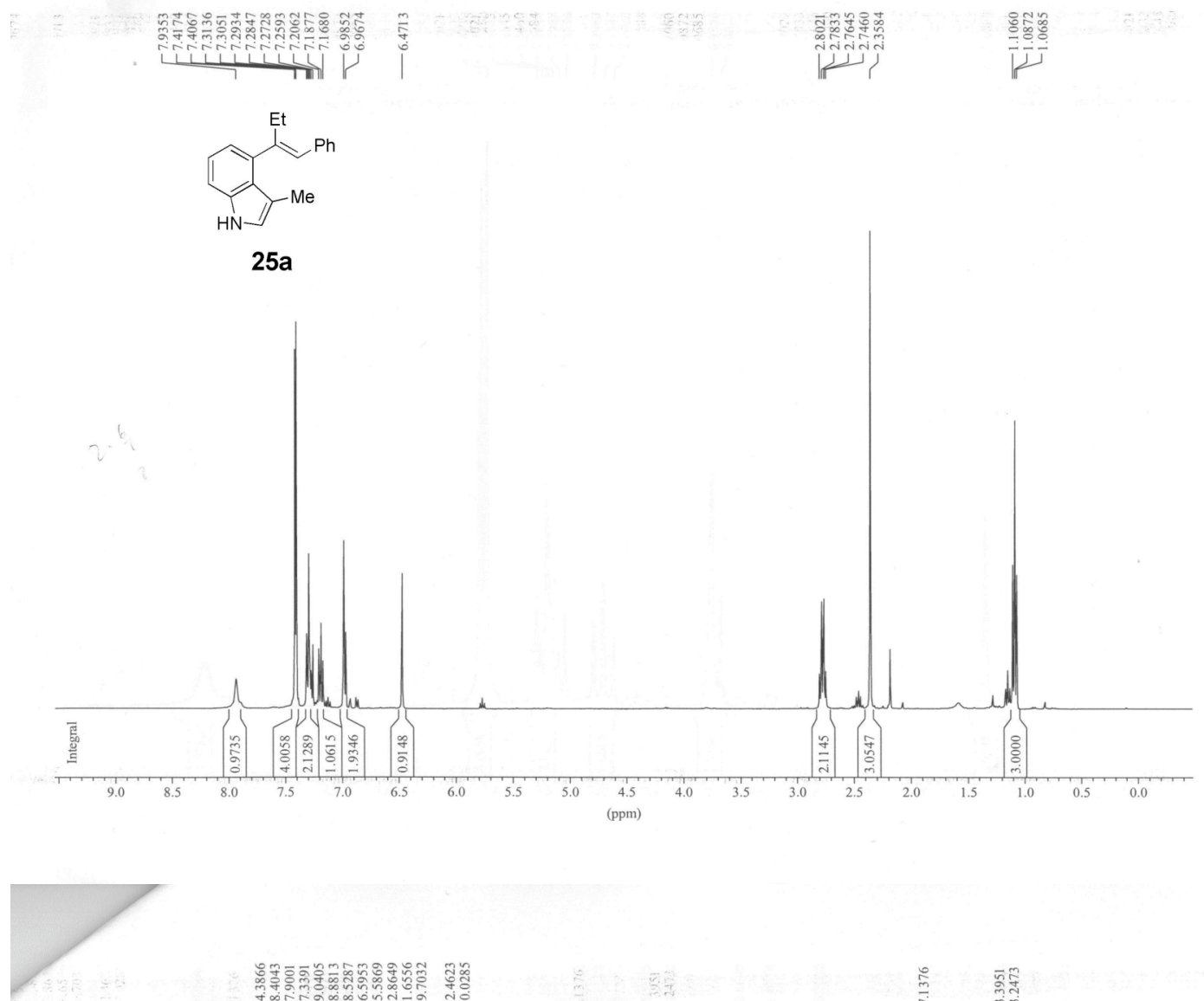

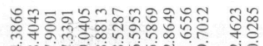

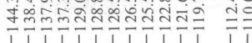

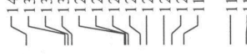

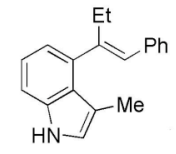

$25 a$

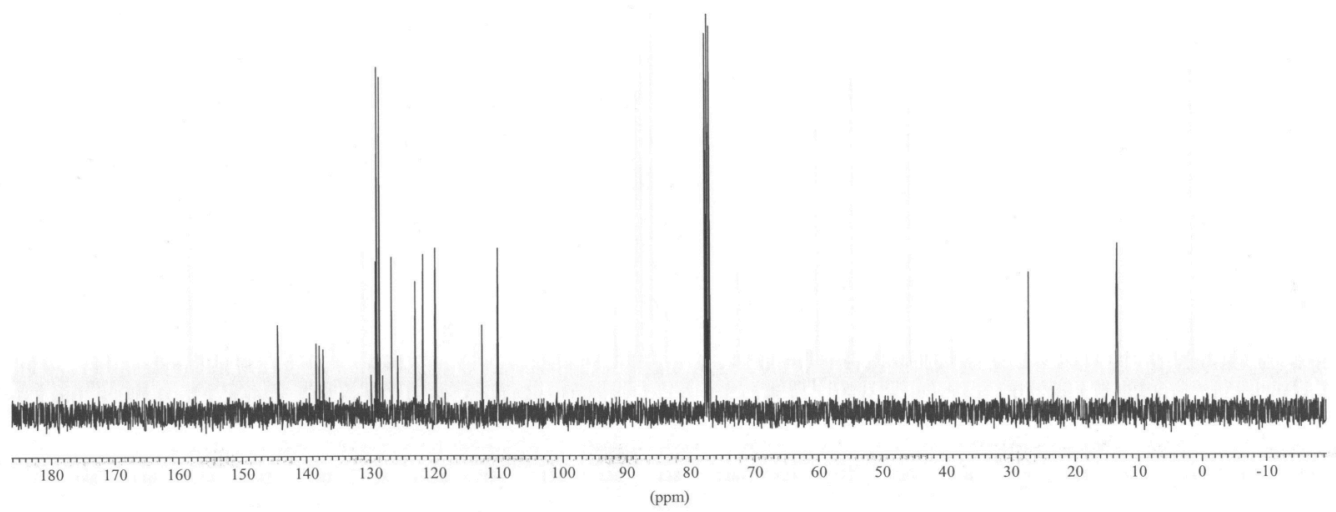

